\title{
AN ANDROID BASED ADVISOR SYSTEM FOR EFFICIENT VEHICLE DRIVING DIRECTIONS
}

\author{
G.Vidhya $^{1}$, V.Ezhilvani ${ }^{2}$, R.Nedunchelian ${ }^{3}$ \\ ${ }^{1} P G$ Student, Department of Computer Science, Saveetha school of engineering, Chennai, Tamil Nadu \\ ${ }^{2} P G$ Student, Department of Computer Science, Saveetha school of engineering, Chennai, Tamil Nadu \\ ${ }^{3}$ Professor, Department of Computer Science, Saveetha school of engineering, Chennai, Tamil Nadu
}

\begin{abstract}
An android based advisor system based on the human intelligence to reach a particular destination at a given departure time. A good routing service is based on routes, traffic and drivers. GPS - equipped taxis are employed as mobile sensors providing the location-based services. Google and Bing maps also provide driving directions is just for user information. A landmark graph is built to estimate the traffic conditions based on certain parameters. Optimal path routing algorithm involves in identifying the optimal route based on the latitude and longitude position of the mobile user to reach a particular destination. The information about the user location is stored in the database a knowledge map is created to update the real time information to the mobile user.
\end{abstract}

Keywords - Global positioning system, Android, Human intelligence. ****

\section{INTRODUCTION}

These days traffic has becoming an important problem in the metro cities, which consumes lot of time to move from one place to another place. The development in the information technology has gained the hope of many people to access and transfer the data anywhere and anytime. Now-a-days the lifestyle of the people is very fast in the current society. The development in the mobile technology has saved the time of people. Android is an open source software based on Linux operating system used in many smartphones and tablets. An android smartphone allows the designer to customize the application into the mobile phones.one of the application in smart mobile phones is GPS(Global Positioning System)which determines the location on the earth.

Travelling is one of the part in our daily life since every day we travel from one place to another place for several reasons.in a metro Politian cities usually consists of large number of taxis and taxi drivers often possess a good knowledge of their area and they follow the most familiar routes to reach different destinations. The most familiar routes are not always the fastest route or shortest route. The real time traffic management has become an important problem in our daily life due to several reasons such as increasing traffic, unexpected calamities, and accidents due to several reasons.

An android based mobileapplication is designed to reduce the time delay of the users and updates the real time formation. The GPS in the mobile phone determines the location of the user based on the latitude and longitude position of the mobile user in this fast living environment. People can take use of this application where people need not spend their time in traffic. The existing navigation service is compared with the information obtained from the previous trips which involves in prediction of travel time and to provide a more popular route to reach a particular destination. The performance of the navigation system is low compared to the taxi driver's route.

Thus, our aim is to make use of our mobile application and updates the real time traffic information with the use of Android operating system to reduce the time delay of the users. An android based system will track the location of the mobile user using the GPS co-ordinates. The real time information updated through the GPS reduces the time delay of the users. In this fast living environment, people will be able to save their time and enjoy their life. The GPS route is more efficient than the Google routes

\section{PROBLEM DEFINITION}

The major problem in our day to day life is traffic congestion .In the existing systemGoogle and Bing maps also provide driving directions for the vehicles. The information provided by the Google and Bing maps is just user information they do not update real time information so people waste their time during traffic and money is wasted on fuels, consumes more money.The smartphones along with mobile sensors gathers the data about the real time traffic conditions. The sensor inbuilt in the mobile phones consumes high processing power and consumes more cost. The data obtained from the co-ordinates determines the speed and time of a user while moving from one source to another destination. The reasons for traffic are due to accidents on the road, failure of signals, and traffic during peak time.The metrics such as route length, travel time, and route popularity are evaluated to compare the performance of the routes suggested by Google. 


\section{PROPOSED SYSTEM}

The user should have an Android based smart phone. This system will be advantageous only for such users. An Android background application is created for the mobile users which involves in capturing the location of the users. The mobile users need to install this application and users are requested to login for obtaining the service. The mobile user need to register the IMEI number and GPS involves in tracking the mobile location of the user while moving from one place to another place the location information is updated and stored in the database.

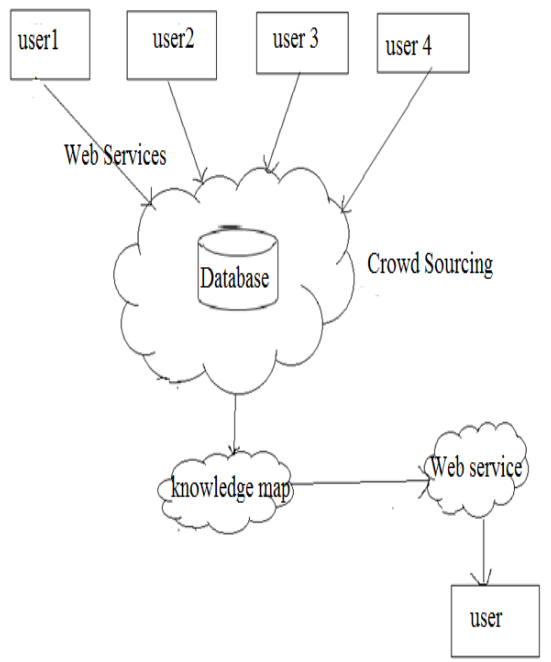

Fig 1: System Architecture

The data needs to be mined from the database whenever a user inquiries for a particular information to reach a particular location. Mining the data from the database involves in the clustering algorithm involves in grouping up of similar objects similarly the most frequently traversed routes is obtained from the GPS loggers. A knowledge map is created from the database to specify fastest route to the user's particular location. A web service is created to expose the knowledge map.an android application involves in providing optimal path to the mobile users.

\section{METHODLOGY}

An Android based advisor system is a dynamic route generation and provides route guidance to the user. The route decision process is alienatedinto two phases, such as before driving and on driving. The routing process of a system

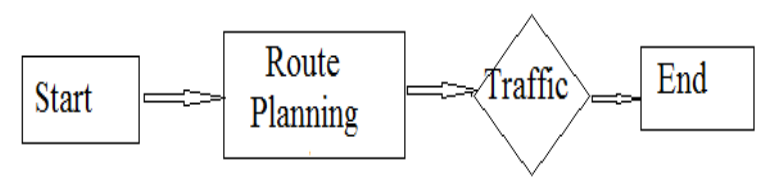

The before driving phase uses the historical data and on driving phase uses the real time data. The current real time data can be generated by using the GPS in the smartphones. Based on the historical data the most frequently traversed routes can be selected by the mobile users. The most frequently routes are shortest path since they are travelled by the several users to reach destination.

\section{ALGORITHM}

An optimal path routing algorithm involves in calculating the path to the mobile users. Start with the GPS co-ordinates such as latitude and longitude where the graph is built.

\section{Optimal Path (Latitude, Longitude)}

Input: a road segment $\mathrm{G}$, a collection of road segment $\mathrm{M}$, list L.

Output:a Graph(date $\&$ time)

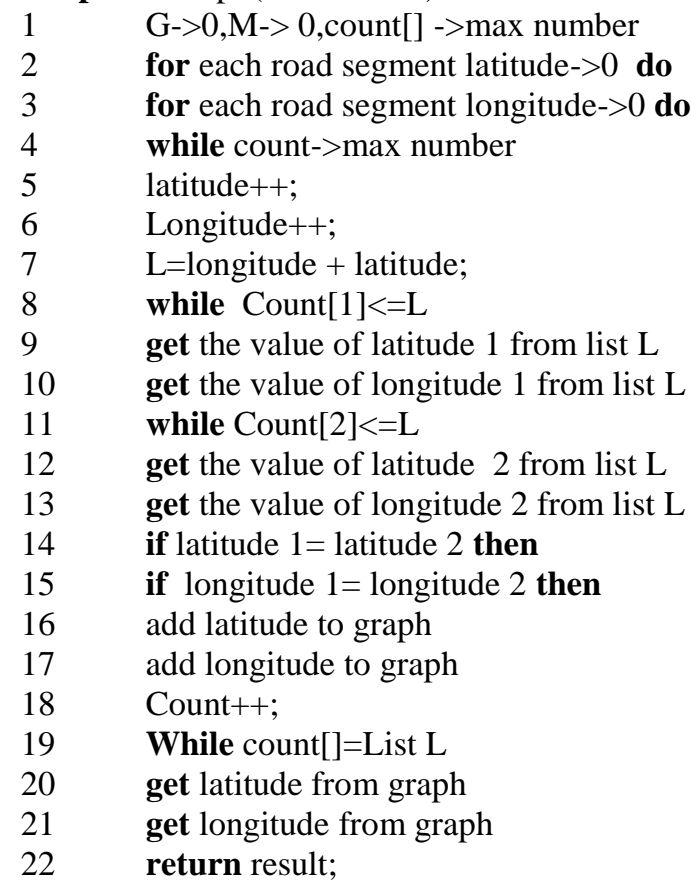

\section{IMPLEMENTATION}

The user enters into the application and specifies the source and destination and checks while traffic is high, and approximately how much time it will take can be calculated. The users IMEI number will be stored in the database. The latitude and longitude position of the user is updated dynamically. The information is updated dynamically to the users of application.

\subsection{Web-Site Designing}

An eye-catching website will be designed using Java and Eclipse software. The website stores the IMEI number of the mobile user in the database.

Fig 2: Navigation System 


\subsection{Calculation of Shortest Path}

Calculation of shortest path will be done by using optimal path routing algorithm. Initially the latitude and longitude position is set to zero and count value is initialized to one and add the latitude to the latitude array and longitude to the longitude array store both the values to the database. The latitude and longitude positions are added to the graph.

\subsection{Determination of Direction}

The coordinates are used to determine the direction of the movement. The latitude north is positive and south is negative. The longitude east is positive and west is negative.

\section{RESULTS AND DISCUSSION}

The time delay may occur due to several reasons such as traffic, accidents etc. Several techniques has been proposed to monitor and control the traffic on the road.an android application is developed using the software where the client server communication takes place through the network. The routing service provided by the current navigation system is compared with the driving behavior of local taxi drivers. The performance of the navigation system is low compared to the taxi driver's route. The sensor inbuilt in the mobile phones consumes high processing power and consumes more cost. The real time traffic management considers the factors such as driving directions, route, traffic, signals etc. So an android application in the mobile updates the real time information so a time delay can be estimated easily to save the time, fuel consumed by the vehicles.

\section{SCOPE AND ITS APPLICATIONS}

Location Based Services and Global positioning System have developed a lot for obtaining the directions, required to the user. Today Google and Yahoo are most familiar names for retrieving the text similarly Location Based Services are familiar for obtaining the directions. The scope of this application is used in metropolitan cities to reduce the traffic. Taxi drivers can use this application for reducing the time delay of the users to reach their destination. It can be useful for public transportation and vehicles. This application will provide free of service. The advantages of this application is less time consumption, less fuel consumption, dynamically information is updated.

\section{CONCLUSIONS}

Thus traffic management system has become very important to save our daily life. An android application implemented in the mobile gathers several information about the user location using the GPS co-ordinates. Several algorithms had been implemented to find the shortest path based on several parameters such as length, time, and route. An optimal path routing algorithm updates the real time information to save timings without time delay. Further research would be done on updating the information from weather forecasting.

\section{REFERENCES}

[1]. J. Yuan, Y. Zheng, C. Zhang, W. Xie, G. Sun, H. Yan, and X. Xie, "T-Drive: Driving Directions Based on Taxi Trajectories," Proc. 18th SIGSPATIAL Int'l Conf. Advances in Geographic Information Systems (GIS), 2010.

[2]. VaidaCeikute, ChristainS.Jensen,"Routing Service Quality - Local Driver Behaviour Versus Routing Services," International Conferences on Mobile Data Management, 2013.

[3]. koustabhdolui, srijani Mukherjee ,soumyakanti data, "traffic status monitoring using smart devices," international conferences on intelligent interactive systems and assistive technologies, 2013.

[4]. J. Yuan, Y. Zheng, C. Zhang, and X. Xie, "An Interactive-Voting Based Map Matching Algorithm," Proc. Int'l Conf. Mobile Data Management (MDM), 2010.

[5]. Y. Zheng, L. Liu, L. Wang, and X. Xie, "Learning Transporta- tion Mode from Raw GPS Data for Geographic Applications on the Web," Proc. 17th Int'l Conf. World Wide Web (WWW), 2008.

[6]. Z. Chen, H.T. Shen, and X. Zhou, "Discovering Popular Routes from Trajectories," Proc. Int'l Conf. Data Eng. (ICDE), pp. 900-911, 2011.

[7]. H. Jeung, M.L. Yiu, X. Zhou, and C.S. Jensen, "Path Prediction and Predictive Range Querying in Road Network Databases," VLDB J., vol. 19, pp. 585-602, Aug. 2010.

[8]. N. Malviya, S. Madden, and A. Bhattacharya, "A Continuous Query System for Dynamic Route Planning," Proc. Int'l Conf. Data Eng. (ICDE), pp. 792-803, 2011.

[9]. Y.Liu, J.Zheng, L.Yan and Y.Xu,"Study on the real time navigation Data model for dynamicnavigation",IGARSS'05, 2005,pp.784-787.

[10]. Sandeep Kumar, Mohammed Abdul Qadeer, ArchanaGupta,'LocationBased Services using Android", proceedings of $3^{\text {rd }}$ IEEE International Conference on Mobile Data Management, Singapore, January 2009.

[11]. Tongyu Zhu, Wang Xiang,"TowardsOptimized Routing Approach for Dynamic Shortest Path Selection in Traffic Networks",2008 International Conference on Advanced Computer Theory and Engineering.

[12]. E.Kanoulas, Y.Du,T.Xia, and D.Zhang, "Finding fastest path on a road network with speed patterns", in proc.ICDE,2006

[13]. Y.Zheng, L.Liu, L.Wan and X.Xie and W.Y.Ma, "GeoLife 2.0: A Location Based Social Networking Service", In proceedings of International conference on Mobile Data Management 2009. 